\title{
INTRODUCCIÓN A LA OPTOELECTRÓNICA (II)
}

\section{INTRODUCTION TO OPTOELECTRONICS (II)}

\section{AUTORES}

Arturo Pérez París: Alumno de la Escuela Politécnica. Universidad de Alcalá arturo.perez@aol.com

\section{CURRÍCULUM VITAE}

Alumno de la Escuela Politécnica de la Universidad de Alcalá de Henares (España). Ingeniero de Soporte Técnico en Kone Elevadores

\section{RESUMEN}

Quizás, cuando empecé a escribir sobre este tema, creí que sería mucho más sencillo. Obviamente me estoy basando en conocimientos existentes y no me estoy inventando nada nuevo. Sin embargo, sí resulta duro realizar un artículo de divulgación breve, que no un libro, por lo que pido disculpas por la posible imagen de falta de rigor que pueda dar al simplificar tanto las cosas. Es preferible que sea así para llegar más fácilmente y a mayor cantidad de lectores, en vez de escribir algo que ni yo mismo sepa de lo que hablo.

\section{PALABRAS CLAVE}

Optoelectrónica - Homenots - Cesar Mataix 


\section{ABSTRACT}

Perhaps, when I started writing on this subject, I thought it would be much easier. Obviously I am not relying on existing knowledge and I'm not inventing anything new. However, it is hard to make a brief popular article, not a book, so I apologize for the possible image of lack of rigor that can give both simplify things. Well it is preferable to get easier and more readers, instead of writing something that even I know what I mean.

\section{KEY WORDS}

Optoelectronics - Homenots - Cesar Mataix

\section{Introducción}

Quizás, cuando empecé a escribir sobre este tema, creí que sería mucho más sencillo. Obviamente me estoy basando en conocimientos existentes y no me estoy inventando nada nuevo. Sin embargo, sí resulta duro realizar un artículo de divulgación breve, que no un libro, por lo que pido disculpas por la posible imagen de falta de rigor que pueda dar al simplificar tanto las cosas. Es preferible que sea así para llegar más fácilmente y a mayor cantidad de lectores, en vez de escribir algo que ni yo mismo sepa de lo que hablo.

Creo que mucha gente, por lo que he visto hasta, ahora no se atreve a escribir por miedo a no dar la talla. No me parece que eso lo deba determinar el autor o un/os critico/s; lo juzgará el lector, a modo individual, bajo su propio criterio. Por ello, 
animo a escribir sin miedo y a expresase libremente con el fin de compartir una parte tangible de nuestra alma: el conocimiento.

Comencemos con el tema que aquí nos ocupa y dejémonos de divagaciones. En esta segunda parte, veremos las diferentes fuentes de luz y la teoría fotoeléctrica. En el estudio de las aplicaciones de la luz es importante conocer las diferentes fuentes luminosas disponibles, con el fin de poderlas utilizar de forma adecuada en los procesos fotoeléctricos.

\section{Fuentes de luz}

La luz se produce hoy por hoy de varias formas, como son:

Combustión: basado en "quemar" una materia ignífuga. Hasta finales del siglo XIX era el único medio de iluminación utilizado por el ser humano.

Incandescencia: emisión de luz por efecto de las altas temperaturas. Hacia 1.878 Edison construyó la primera lampara eléctrica de aplicación práctica. Al paso de la corriente eléctrica a través de un filamento se produce calor y una radiación luminosa.

Descarga a través de un gas: esta fuente de luz está basada en la emisión de determinadas longitudes de onda, originadas por la energía perdida por los electrones libres en un gas ionizado, cuando son de nuevo atrapados por el átomo (las frecuencias de estas radiaciones suelen estar en el rango del ultravioleta).

Fluorescencia: si bien este método es similar al anterior, añadiremos que las radiaciones ultravioletas obtenidas en las lámparas de descarga, al incidir sobre los 
polvos fluorescentes que tapizan las paredes de la lámpara, inducen radiaciones de estos materiales en el rango de frecuencias de la luz visible.

Electroluminiscencia: este fenómeno de emisión de luz es el más moderno de todos y todavía se halla en desarrollo. Se basa en la excitación, o aumento de la energía, de los electrones de una sustancia, los cuales, al retornar a su nivel normal irradian una energía luminosa en el espectro visible.

Como todos sabemos, el átomo se encuentra constituido por un núcleo y los electrones que orbitan a su alrededor con sus diferentes niveles energéticos (teoría de bandas):
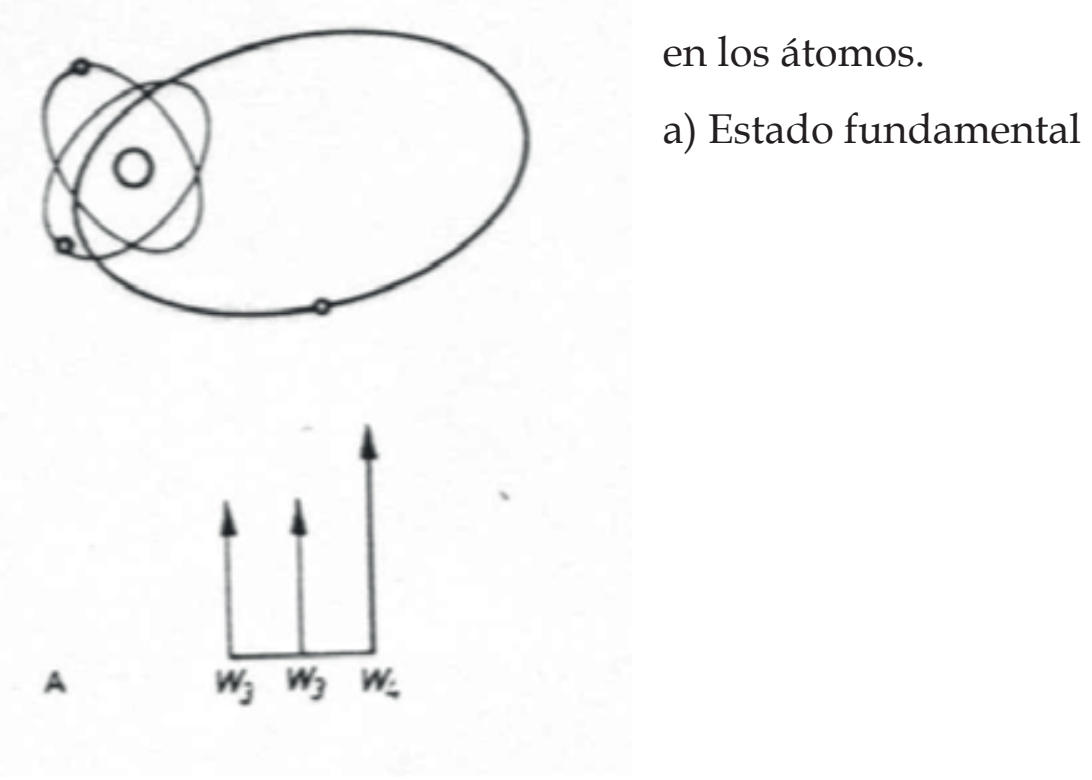

Producción de luz por excitación de electrones en los átomos.

a) Estado fundamental

Si un electrón adquiere una energía adicional, por choque con otros, o debida a un campo eléctrico, o por otras razones, pasará a ocupar una órbita más alejada del núcleo: 


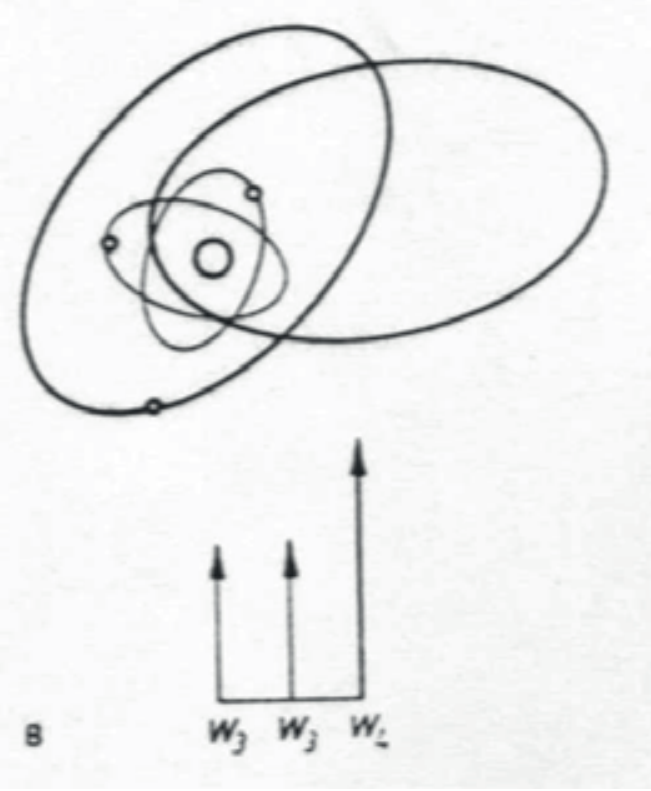

Producción de luz por excitación de electrones en los átomos.

b) Estado excitado

En todos los fenómenos físicos, el estado de equilibrio es el de menor energía posible; por ello, el electrón excitado tenderá a retornar a su estado inicial, que se encuentra vacío. Al hacerlo, la energía en exceso de que dispone será radiada con una frecuencia o longitud de onda proporcional a aquella:

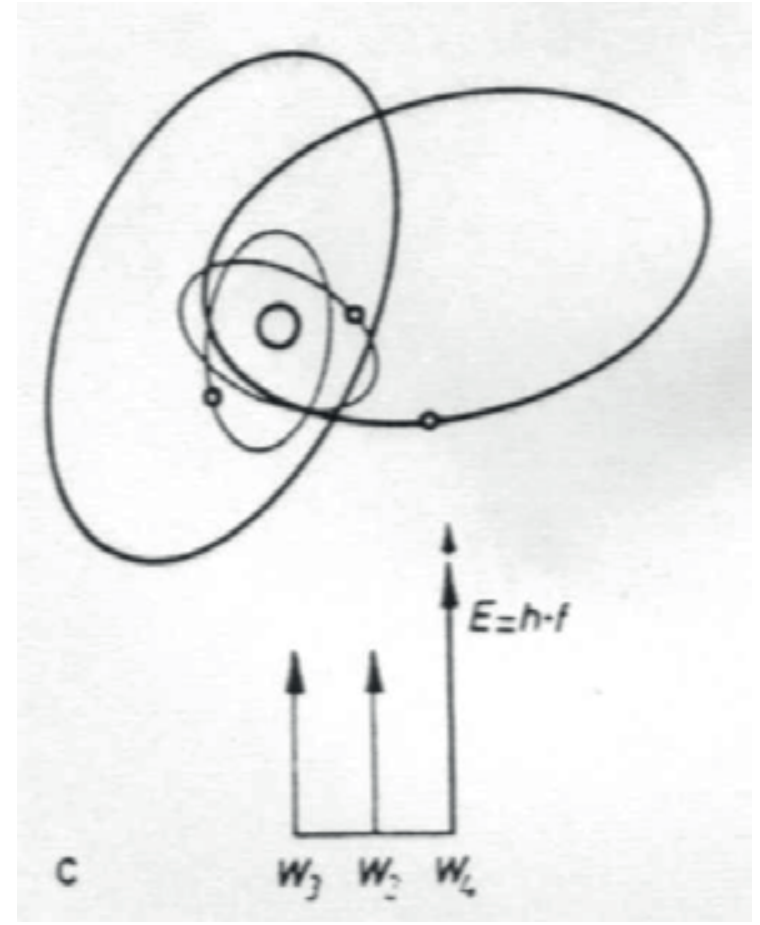

Producción de luz por excitación de electrones en los átomos.

c) Vuelta al estado fundamental 
A la energía desprendida se la denomina fotón o cuanto de luz, ya que los niveles energéticos que puede ocupar un electrón en la corteza del átomo corresponden a múltiplos enteros de una cantidad de energía mínima fundamental (cuantos). El proceso que se ha descrito es reversible, esto es, si un átomo es irradiado por una energía luminosa, los electrones de sus últimas capas se excitarán pasando a ocupar órbitas de mayor energía, pudiendo llegar incluso a abandonar el átomo, quedando libres en el medio o emigrar al exterior del material (efecto fotoeléctrico). El proceso directo de producir radiaciones luminosas se suele conocer con el nombre de electroluminiscencia y la producción de portadores de carga (electrones) por efecto de la luz, se conoce con el nombre de fotoemisividad.

\section{Fotoelectricidad}

Supóngase que se irradia un metal con energía luminosa, compuesta por fotones de todas las frecuencias del espectro visible, siendo la energía de cada fotón:

$\mathrm{W}=\mathrm{hf}$

con

W: energía del fotón entrante.

h: constante de Plank.

f: frecuencia de la radiación entrante.

Cuanto mayor sea la frecuencia, tanto mayor será la energía; por lo que las radiaciones violetas y ultravioletas son de mayor energía que las rojas, que pertenecen al otro extremo de la banda. Así las cosas, los electrones de algunos átomos adquirirán la energía de estos fotones, pasando a ocupar órbitas más alejadas del núcleo y aumentando con ello la velocidad de su movimiento. Si un electrón absorbe la suficiente energía, alcanza así la suficiente velocidad como para escapar de 
las fuerzas de atracción del metal. Esta es la propiedad conocida como fotoemisividad:

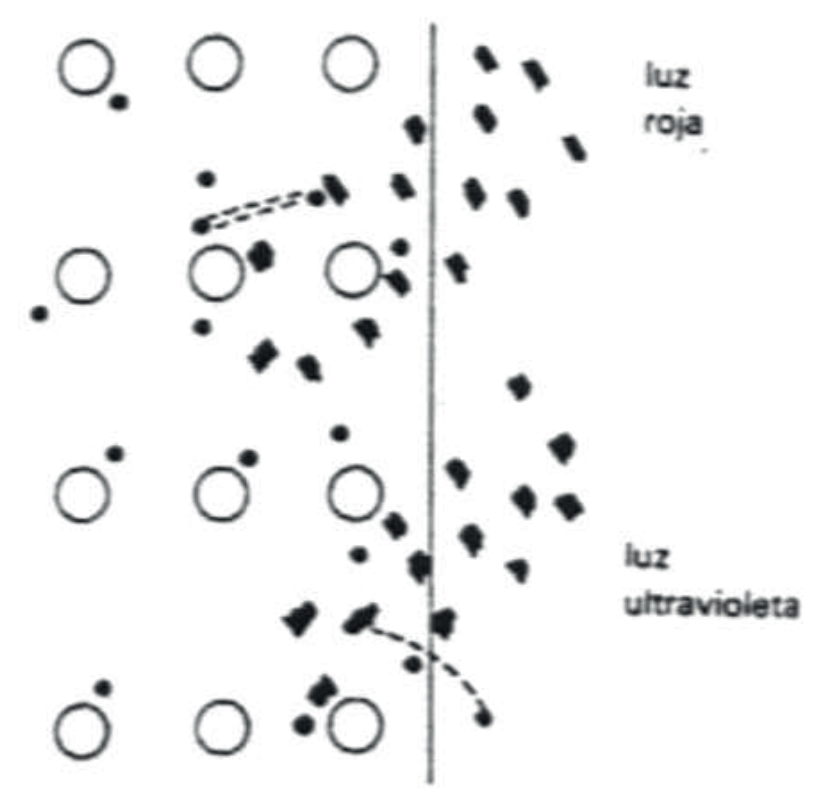

Fotoemisión de los metales

Obviamente, como todo en la naturaleza, hay un límite para la frecuencia a la que se puede dar el proceso de emisión electrónica, que depende de cada material. Por debajo de ella no es posible extraer electrones de un metal. Dicha frecuencia se llama frecuencia umbral y es aquella que marca el límite de fotoemisión en un metal concreto. Nótese que la fotoemisividad no es función del flujo luminoso irradiado sobre el metal y sí de la frecuencia de los fotones.

Cuando un fotón de baja energía (rojo) incide sobre un electrón, la energía de éste, como ya se dijo, será pequeña con lo que no conseguirá producir la fotoemisividad; aunque sí aumenta su velocidad de desplazamiento por el metal por habérsele aumentado su energía cinética y potencial. Por esa razón, puede pasar a la banda de conducción desde la de valencia, aumentando la cantidad de portadores de la primera y por ello la conductividad del material: 


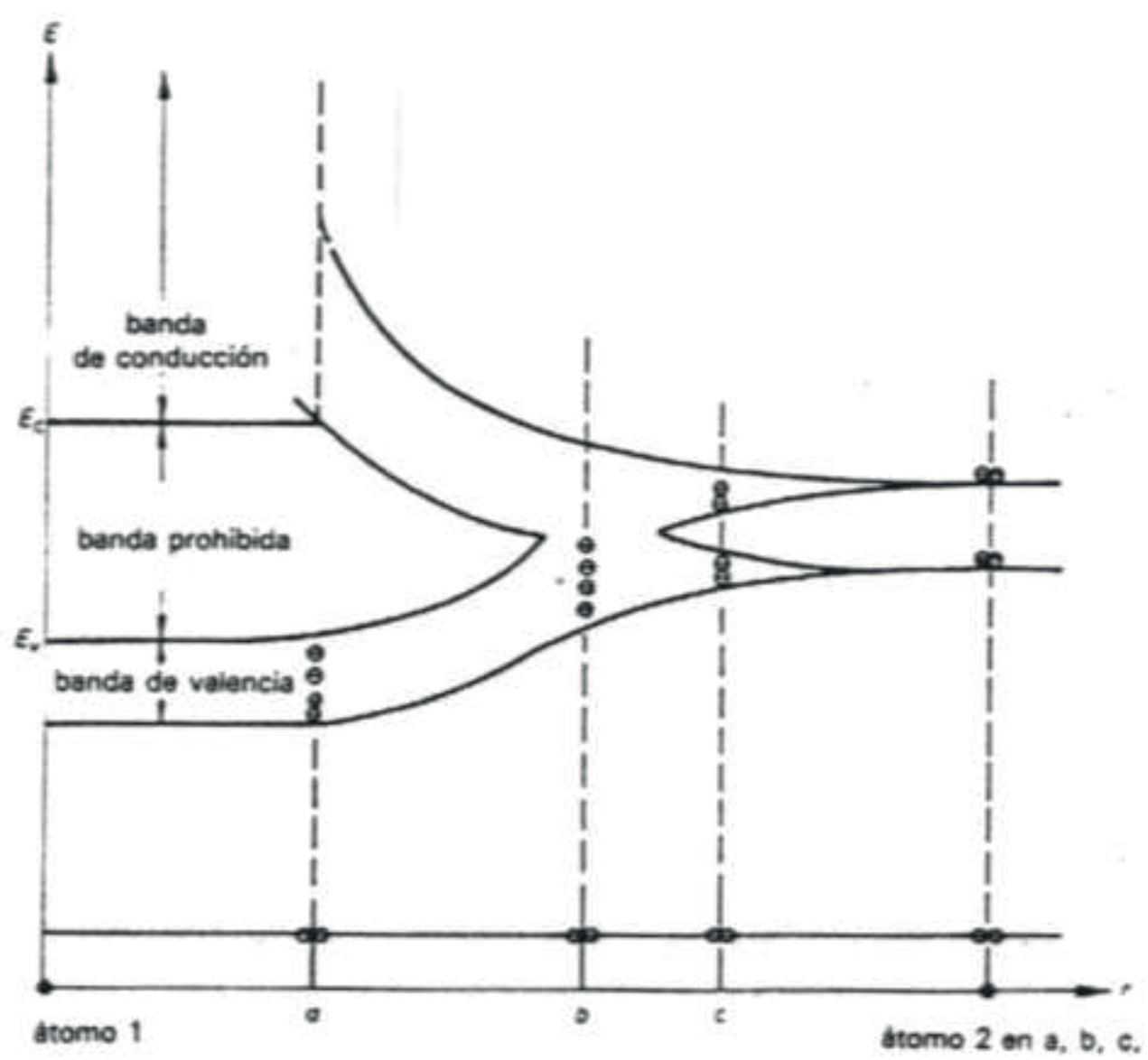

Bandas de energía de los semiconductores, teniendo presente la posición relativa de un átomo próximo.

Mediante los efectos aquí relacionados constituiremos los dispositivos fotoeléctricos, que en sí es el objetivo que perseguimos, es decir, la emisión de luz en sus diferentes longitudes de onda y la captación de éstas para uso eléctrico.

El primer elemento a presentar son las fotocélulas. Por tales se designan los dispositivos fotoeléctricos, en los cuales la emisión de electrones se produce por efecto de la energía que la luz puede suministrar a los electrones más externos (podríamos decir superficiales) de los átomos. La energía de la radiación electromagnética de la luz (fotones) depende de la frecuencia de radiación, como ya hemos mencionado. Cuando un fotón llega a un metal y es absorbido por un 
electrón, la energía cinética de esta último aumenta bruscamente, pudiendo hacer que el electrón salga del metal y produciéndose así la fotoemisión:

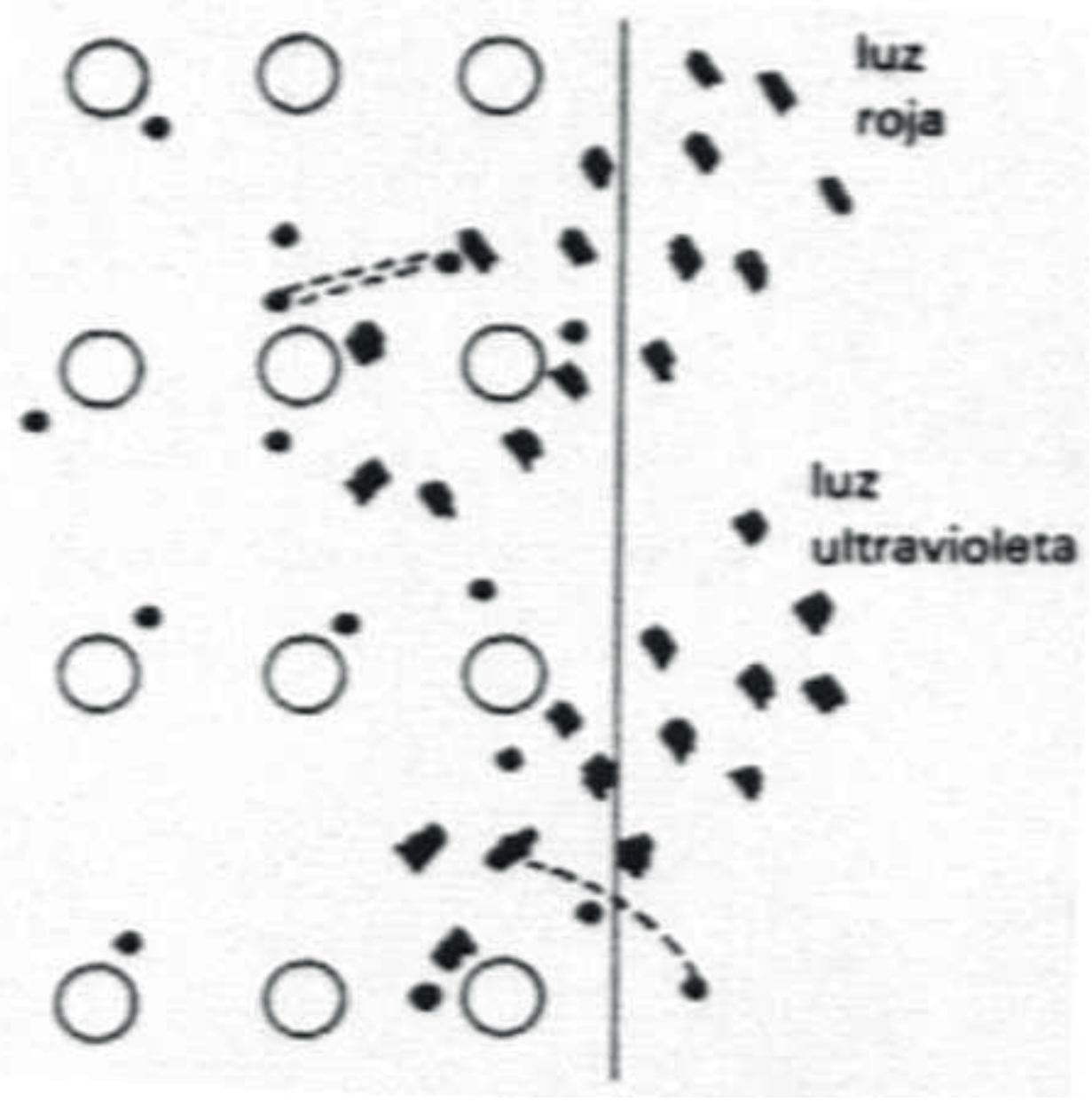

Fotoemisión en los metales

Dentro del espectro de longitudes de onda correspondientes a la radiación luminosa, la región fotosensible no es igual para todos los metales, pero la mayoría se halla en la región ultravioleta. Llegado a este punto debemos introducir un concepto esencial dentro de los dispositivos fotoeléctricos. Éste es la sensibilidad, y se define en principio como la relación entre el número de electrones emitidos por el material fotosensible y la longitud de onda de la radiación luminosa aplicada: 


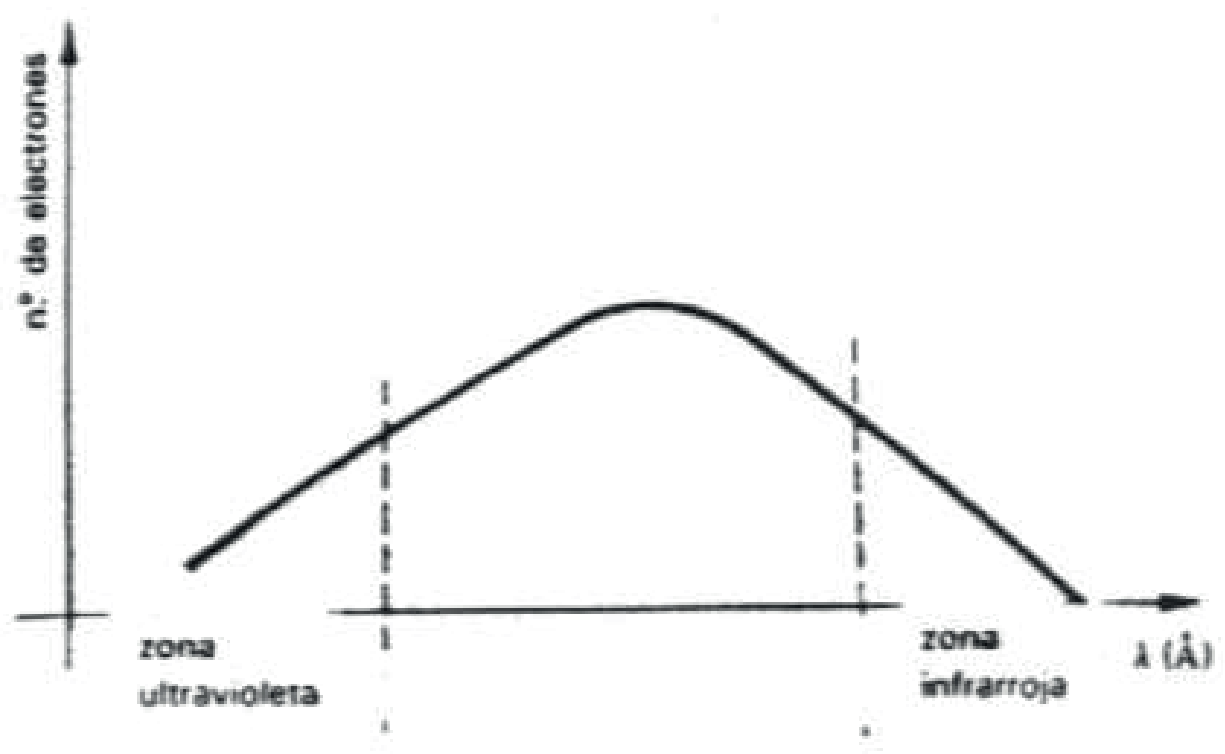

Curva de sensibilidad

De lo expuesto hasta ahora podría pensarse que el mayor número de electrones debe conseguirse con la luz ultravioleta, ya que por ser su longitud de onda menor, o frecuencia mayor, resulta mayor la energía de sus fotones; sin embargo no es así realmente, ya que cada material presenta una frecuencia característica, a la cual su emisión es máxima:

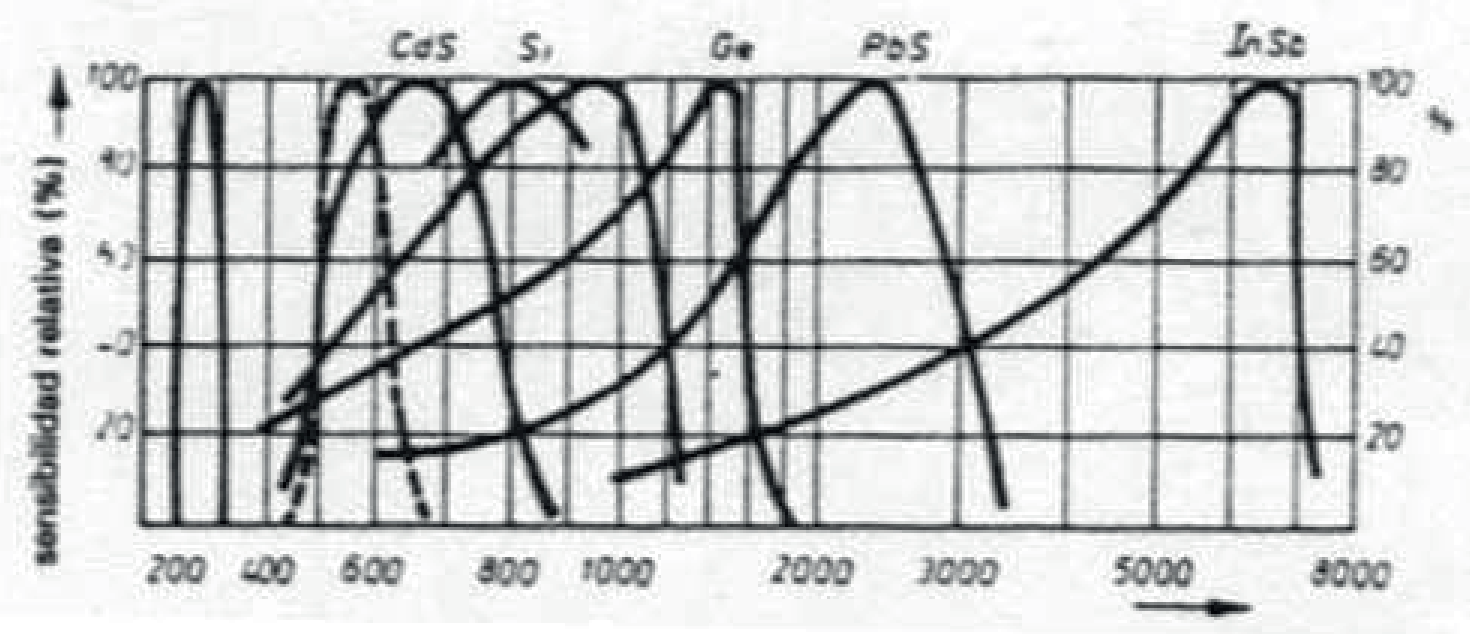

Sensibilidad espectral relativa 
Otro efecto fotoeléctrico interesante, aunque propio de los semiconductores, es el fotovoltaico en las uniones semiconductoras. Ésta se debe a que la energía de la radiación luminosa que incide sobre ella rompe los dobles enlaces del cristal, tal y como se muestra a continuación:

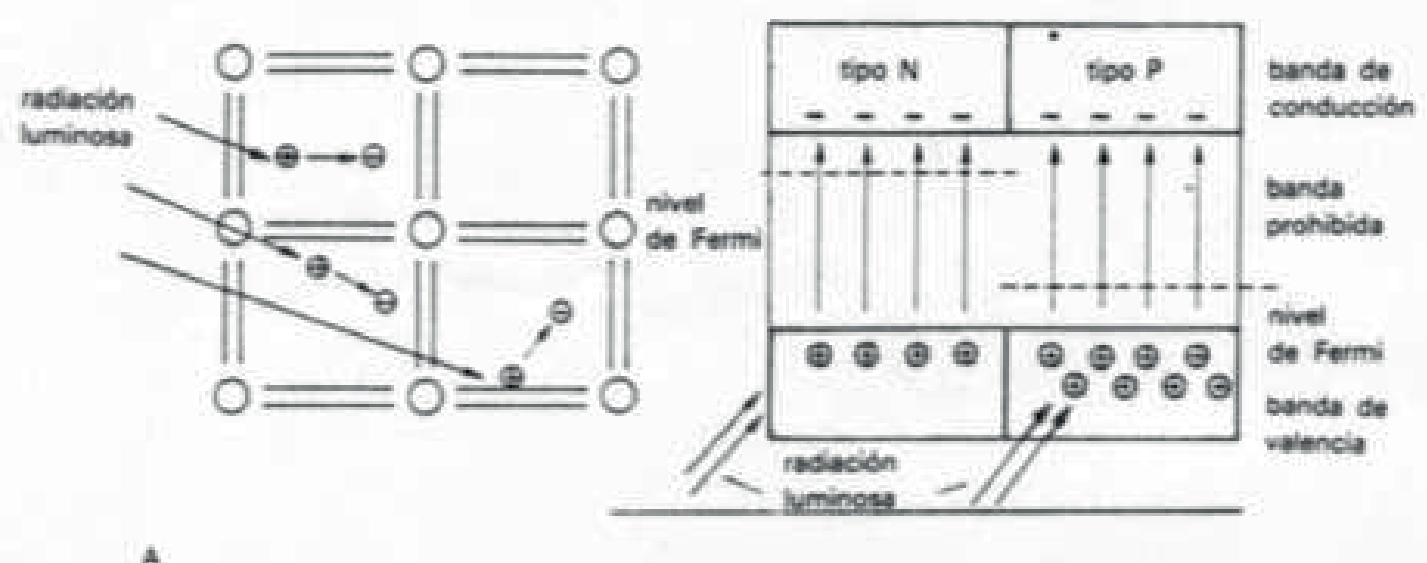

Efecto fotovoltaico: Formación del efecto fotovoltaico en una unión semiconductora

pero su efecto es diferente, según se trate de un material tipo $\mathrm{N}$ o un tipo $\mathrm{P}$ produciendo exceso de electrones en el tipo $\mathrm{N}$ y exceso de huecos en el tipo P. Este desequilibrio de cargas genera una tensión y una corriente proporcionales a la diferencia de potencial en la barrera de la unión. Ese potencial, a su vez, depende de la proporción de dopado de las regiones N y P conjugándose como coeficiente de absorción con la longitud de onda de la radiación luminosa: 


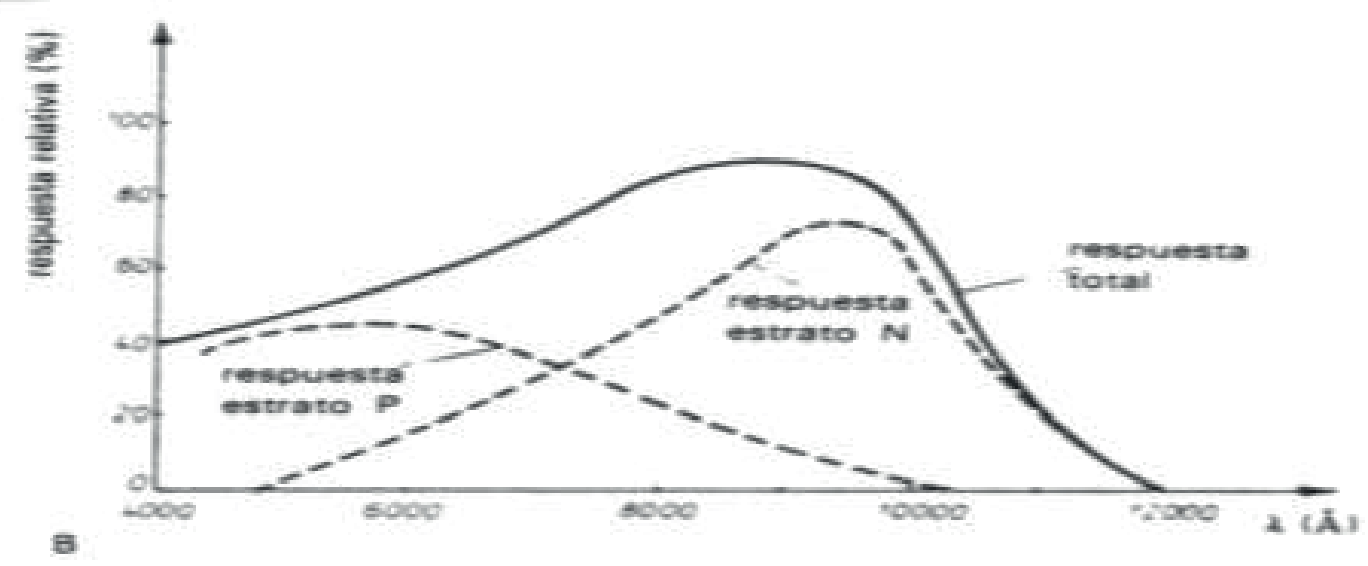

Efecto fotovoltaico: Variación del número de portadores en función de la longitud de onda de la radiación incidente

Con esto concluimos la segunda parte de este artículo. Algo más corto que el anterior pero quizás más denso y, posiblemente, no tan básico. Recomiendo que, si algo no está claro, se recurra a cualquier libro de Física como el Tippler, el Alonso y Finn o uno equivalente. En la próxima entrega presentaremos los dispositivos fotoelectrónicos que serán la estrella de este trabajo. Les espero entonces . 